\title{
A Novel Method to Verify Gamma Knife Radioactive Source Count
}

\section{Hsein-Tang Tu1,2, Cheng-Siu Chang,,4, Chun-Yi Chuang4, Cheng-Wei Huang5*, Wei-Jr Li', Tzu-Chin $\mathrm{Wu}^{3,4}$, Fu-Yu Feng ${ }^{7}$, Zih-Ping Ho ${ }^{8}$, Chuan-Fu Huang ${ }^{1}$}

${ }^{1}$ Gamma Knife Center, Chang Bing Show Chwan Memorial Hospital, Taiwan

${ }^{2}$ Division of Pulmonary Medicine, Department of Internal Medicine, Chung-Shan Medical University Hospital, Taiwan

${ }^{3}$ Departments of Neurosurgery, Chung-Shan Medical University Hospital, Taiwan

${ }^{4}$ School of Medicine, Chung-Shan Medical University, Taiwan

${ }^{5}$ Department of Internal Medicine, Kaiser Permanente Los Angeles Medical Center, Los Angeles, USA

${ }^{6}$ Gamma Knife Center, China Medical University Hospital, Taiwan

${ }^{7}$ Department of Medical Imaging, Show Chwan Memorial Hospital, Taiwan

${ }^{8}$ Research Assistant Center, Show Chwan Memorial Hospital, Taiwan

Email: *charliehuang7@gmail.com

How to cite this paper: Tu, H.-T., Chang, C.-S., Chuang, C.-Y., Huang, C.-W., Li, W.-J., Wu, T.-C., Feng, F.-Y., Ho, Z.-P. and Huang, C.-F. (2018) A Novel Method to Verify Gamma Knife Radioactive Source Count. Technology and Investment, 9, 154-160.

https://doi.org/10.4236/ti.2018.93011

Received: March 13, 2018

Accepted: August 10, 2018

Published: August 13, 2018

Copyright $\odot 2018$ by authors and Scientific Research Publishing Inc. This work is licensed under the Creative Commons Attribution International License (CC BY 4.0).

http://creativecommons.org/licenses/by/4.0/

\begin{abstract}
Background: Regulation requires periodic reconfirmation of the number of radioactive sources in Gamma Knife (GK). To comply with regulations, the total dose output (TDO) method, originally designed to verify amount of radiation received by patients, was adopted as a tool for source security. However, we suspect the TDO method may be unreliable in detecting small numbers of missing sources given that a $2 \%$ margin of error is allowed. We propose an alternate method using Gafchromic films to identify number of sources present. Method: To test the efficacy of the TDO method, we simulated real-life source loss by plugging up to 5 sources. TDO after plugging 0 , $1,2,3,4$, and 5 sources were measured using GK 4C. Percentage change for each additional source plugged was computed. We then proceeded to test the film method. For GK 4C, 2 Gafchromic RTQA2 single sided films measuring $10 \times 10$ inch were first halved to $5 \times 10$ inch films. Four $5 \times 10$ inch films were then adhered to the inner surface of the $18 \mathrm{~mm}$ collimator. For GK Perfexion, 2 Gafchromic EBT3 double sided films measuring $10 \times 12$ inches were combined and rolled into a cone film and adhered to the collimator during installation. In both cases, the films were exposed to 0.5 Gy. Results: TDO was inversely related to the number of source plugged. TDO decreased by more than $2 \%$ after 5 plugs. The film method was able to outline all 201 and 192 Co sources for GK 4C and Perfexion respectively. Conclusion: A single lost source is hard to detect using the TDO method given that at least 5 sources had to be lost before the change in TDO will exceed the margin of
\end{abstract}


error $( \pm 2 \%)$. The film method bypasses this limitation and offers an easy alternative to accurately obtain the number of sources.

\section{Keywords}

Source, Security, Gamma Knife, Radiosurgery

\section{Introduction}

The Legislative Yuan Atomic Energy Council Taiwan has mandated that all hospitals containing category 1 and 2 radioactive sources have a security plan [1]. Part of the mandate includes a nuclear source count and periodic reconfirmation of the number of sources. To ensure the security of Gamma Knife (GK) radioactive sources, the total dose output (TDO) method is frequently used as an assessment method [2]. However, the TDO method was originally designed to verify the amount of radiation received by patients and a $2 \%$ margin of error allowed. If only TDO was observed, a small number of missing sources may not be identified as the resulting TDO may be within the margin of error. We sought to examine the efficacy of the TDO method in a radioactive source loss scenario. We ultimately propose a complementary and possibly alternate method using Gafchromic films to identify the number of sources present in GK 4C and Perfexion [3].

\section{Materials and Methods}

\subsection{TDO Method}

We first tested the efficacy of the TDO method. We simulated real-life source loss by plugging a number of sources. Per regulations, the TDO was measured 10 times before each plugging test. Measurements of total dose output after plugging with $0,1,2,3,4$ and 5 were performed using model $4 \mathrm{C}$. We measured TDO for 3 consecutive times for $0,1,2,3,4$, and 5 plugs and computed the average value. Percentage change for each additional source plugged was also computed.

\subsection{Film Method}

We proceeded to test the film method using Gafchromic films. Gafchromic films are radiation sensitive film that can be used for quality assurance. RTQA2 films can detect radiation dosage from $0.02 \mathrm{~Gy}$ to $8 \mathrm{~Gy}$. EBT3 films can detect radiation dosage from $0.001 \mathrm{~Gy}$ to $10 \mathrm{~Gy}$. Areas exposed to $0.5 \mathrm{~Gy}$ through the hole will turn black, allowing for radiation source count.

\subsubsection{Model 4C (201 Co Sources)}

Two RTQA2 single sided films measuring $10 \times 10$ inch were halved into $5 \times 10$ inch films. Four $5 \times 10$ inch films were then adhered to the inner surface of the $18 \mathrm{~mm}$ collimator (Figure 1). Verification that the film covered all holes was 


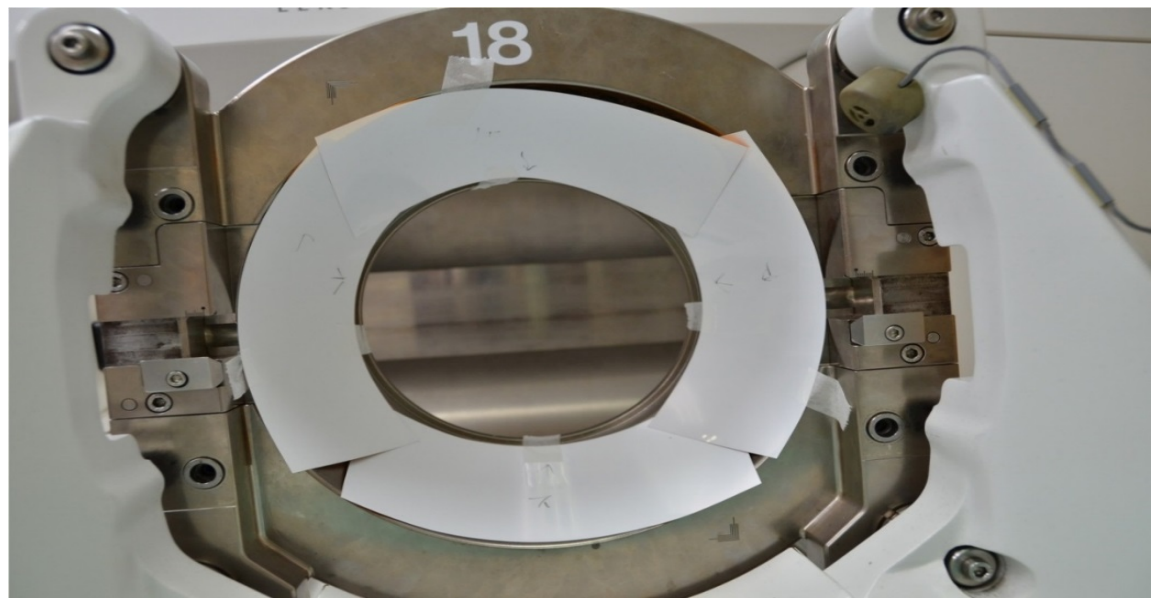

Figure 1. Adhere four $5 \times 10$ inch films to the inner surface of the $18 \mathrm{~mm}$ Collimator (Source: authors).

done before radiation exposure was initiated. The time needed for each source to release 0.5 Gy was first calculated by the daily output rate of the GK unit (3 Gy/min) and number of sources (201). After radiation exposure, we then obtained the total Co source count by counting the number of black circles on the film.

\subsubsection{Perfexion (192 Co Sources)}

Due to the internal nature of the collimator, two EBT3 double sided $10 \times 12$ inch films were trimmed and overlapped to form a single cone film in the center. Coordinates X, Y, and $\mathrm{Z}$ were then set to $(100,100,100)$ and clearance position reached to check for collision (Figure 2). The time needed for each source to release 0.5 Gy was first calculated by the daily output rate of the GK unit (1.23 $\mathrm{Gy} / \mathrm{min}$ ) and number of sources (192). After radiation exposure, we then obtained the total Co source count by counting the number of black circles on the film.

\section{Results}

TDO with $0,1,2,3,4$, and 5 plugs were $10.45 \mathrm{nC}, 10.4 \mathrm{nC}, 10.35 \mathrm{nC}, 10.3 \mathrm{nC}$, $10.25 \mathrm{nc}$ and $10.2 \mathrm{nc}$ respectively. TDO change exceeded $2 \%$ after 5 plugs (Table 1 ). In GK $4 \mathrm{C}$, the time needed for $0.5 \mathrm{~Gy}$ was 33 minutes and the film method outlined 201 Co sources (Figure 3). In GK Perfexion, the time needed for 0.5 Gy was 83 minutes and the cone film outlined 192 Co sources (Figure 4).

\section{Discussion}

Following the September 11 attacks, there has been an increase in both the number of terrorism attacks and different methods [4]. In January 2003, British intelligence discovered a terrorism nuclear waste bomb blueprint. Many terrorists have targeted hospital nuclear sources in hopes to take advantage of a hospital's less stringent security. Terrorists with minimal training and no shielding 


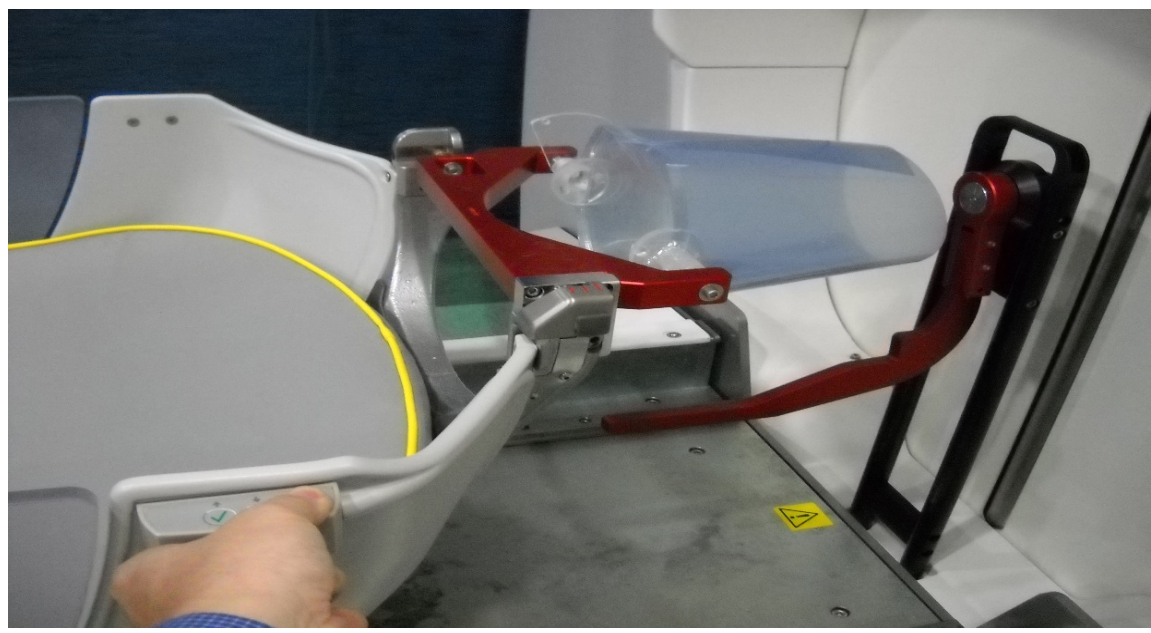

Figure 2. Cone shape film model for Pefexion, two Gafchromic EBT double sided films $10 \times 12$ inch partially overlapped then became cone film similar to collimator shape. Coordinates X, Y, Z $(100,100,100)$ were set and clearance position reached to check collision. (Source: authors).

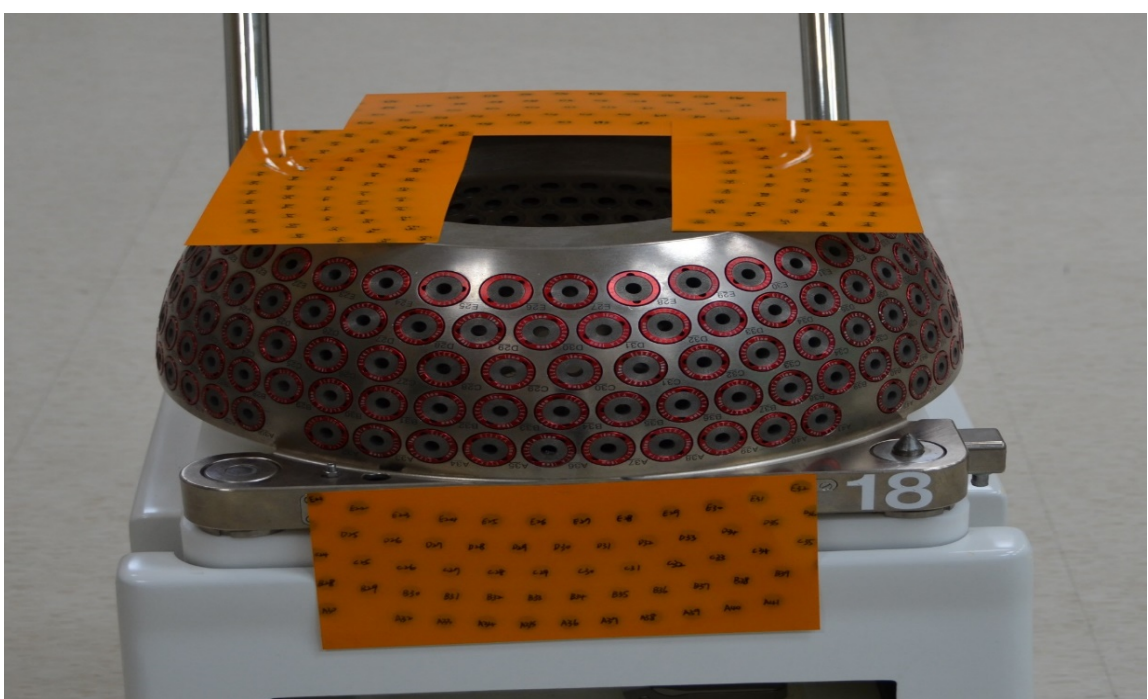

Figure 3. 201 cobalt source detected on the film (Source: authors).

Table 1. Measurement of total dose output after plugging from 0 to 5.

\begin{tabular}{ccc}
\hline Plugs number & Average total dose output $(\mathrm{nC})$ & Output decrease $(\%)$ \\
\hline No plug & 10.45 & $0 \%$ \\
1 plug & 10.4 & $-0.5 \%$ \\
2 plug & 10.35 & $-1.0 \%$ \\
3 plug & 10.3 & $-1.4 \%$ \\
4 plug & 10.25 & $-1.9 \%$ \\
5 plug & 10.2 & $-2.4 \%$ \\
\hline
\end{tabular}

can obtain nuclear sources and create nuclear dirty bombs [5]. The cost of even one single radioactive source can be staggering, as evident by the Goiânia inci- 
dent [6]. To mitigate this risk and protect people against radiation exposure, The International Atomic Energy Agency and the World Institute for Nuclear Security have lead international training efforts to promote training and competence amongst practitioners [7] [8].

In our study, we had 0 to 5 sources plugged to simulate the degree of change in TDO when different numbers of Co sources are lost. At least 5 sources had to be lost before TDO change exceeded the $2 \%$ margin of error allowed by regulations $(-2.4 \%)$ (Figure 5). This simulation suggests that TDO is not a reliable way to assess source security. This leaves Co source count as the best alternative, and our film method allows this to be done with ease.

It is important to note that the collimator of GK Perfexion, unlike that of GK B, C, 4C, is installed inside. Therefore, instead of being able to adhere RTQA2 films to the inner surface of the collimator like in previous generations, we had

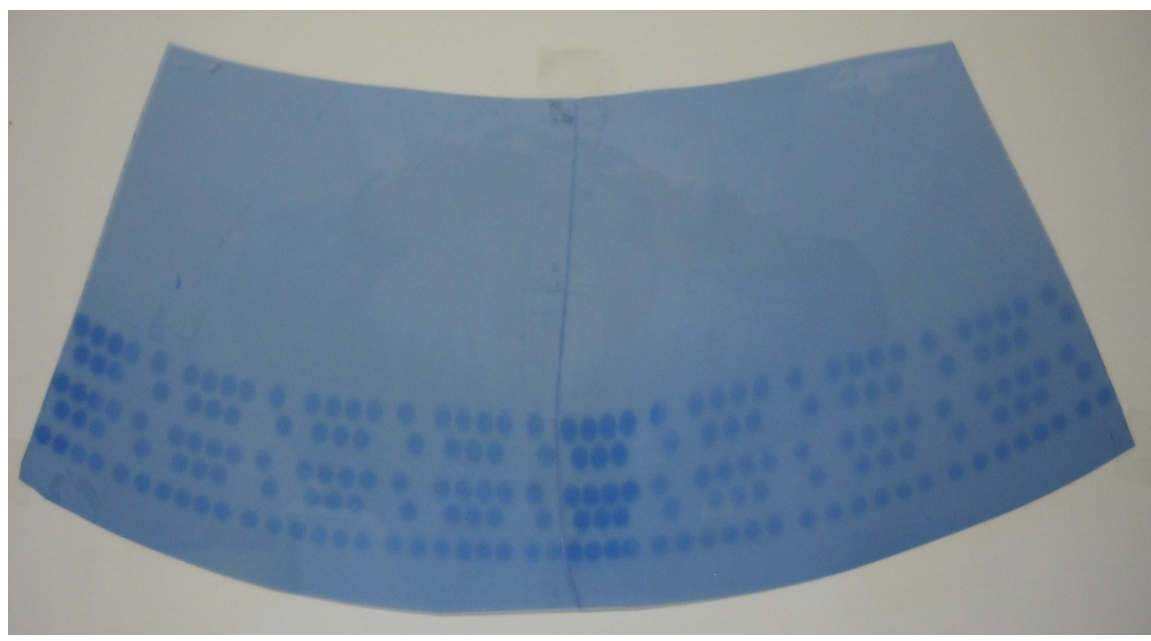

Figure 4. 192 cobalt source detected on the film ( $8 \mathrm{~mm}$ collimator) (Source: authors).

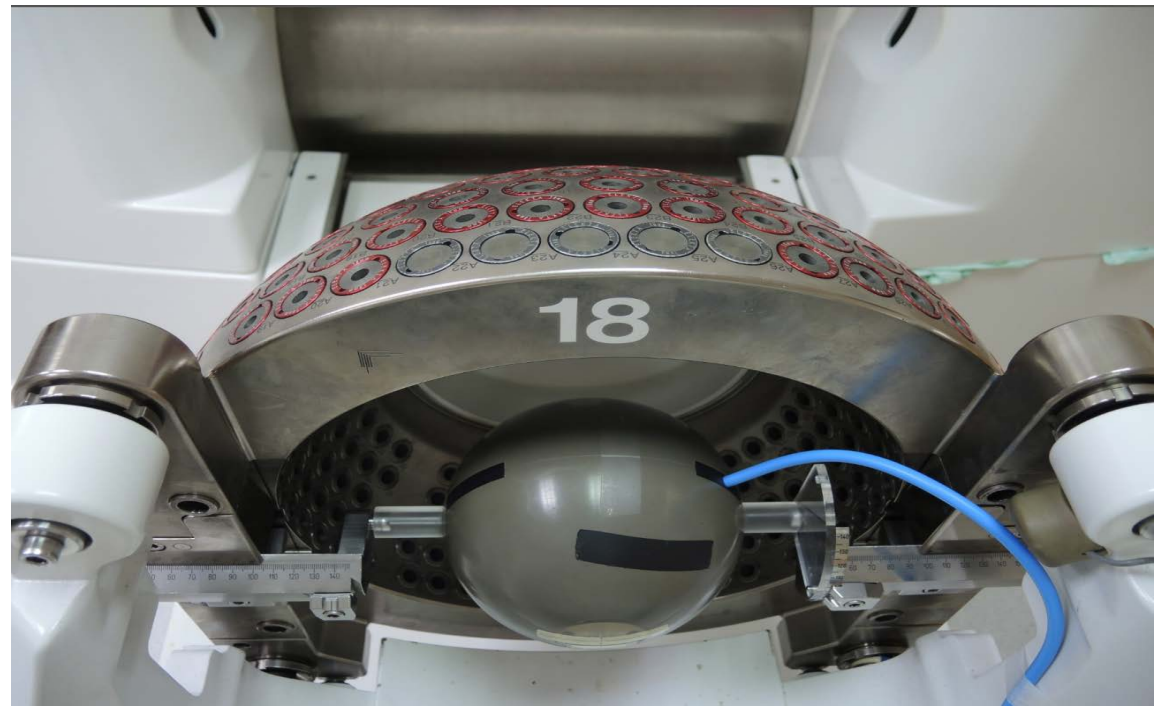

Figure 5. Use five plugs simulate loss 5 Cobalt sources for measurement total dose output. (Source: authors). 
to design a plastic cone to hold the film in place without overlapping to avoid double counting. Other than this minor modification, the approach is largely similar to that of other models.

A limitation of our method is time. While it only took 33 minutes for $0.5 \mathrm{~Gy}$ in model 4C, it took 88 minutes with Perfexion because the sources have decayed for more than one half-life (5.26 years). The time required could be even further extended as the sources further decay over time. To overcome this issue however, one may consider switching to a higher sensitivity film.

In addition, our method may not be able to identify real-time source loss. Even if lost sources are identified at the time of measurement, the source loss could have occurred any time between the current and last measurement. While the frequency of measurements must be balanced with time, the greatest risk of source security is when insiders such as staff or maintenance personnel are associated with the theft. Therefore, our method may be of particular value when employed between the hand-off among staff and maintenance personnel. At our institution, we take measurements quarterly or whenever source related maintenance is performed.

\section{Conclusion}

Regulation compliance and radioactive source security has become an integral process for many healthcare centers. We propose a low cost and easy to use film method that accurately assesses source count regardless of Gamma Knife models. Our approach is practical not only because of its low cost and high efficacy, but also because it only requires a onetime adoption. With increased scrutiny over radioactive source security and concern for global terrorism, our method not only benefits institutions but may also help mitigate the societal costs associated with terrorism acts.

\section{Conflicts of Interest}

The authors declare no conflicts of interest regarding the publication of this paper.

\section{References}

[1] Huang, C.-L. (2012) Environmental Justice and Public Participation: A Case Study of Nuclear Waste Management and Policy in Taiwan. Newcastle University, Newcastle.

[2] Steiner, L., Yen, C.P., Jagennathan, J., Schlesinger, D. and Steiner, M. (2009) Gamma Knife: Clinical Aspects. Textbook of Stereotactic and Functional Neurosurgery, 198, 1037-1086. https://doi.org/10.1007/978-3-540-69960-6_66

[3] Kutcher, G.J., Coia, L., Gillin, M., et al. (1994) Comprehensive QA for Radiation Oncology: Report of AAPM Radiation Therapy Committee Task Group 40. Medical Physics, 21, 581-618. https://doi.org/10.1118/1.597316

[4] Lubenau, J.O. and Strom, D.J. (2002) Safety and Security of Radiation Sources in the Aftermath of 11 September 2001. Health Physics, 83, 155-164. https://doi.org/10.1097/00004032-200208000-00001 
[5] Barnaby, F. (2005) Dirty Bombs and Primitive Nuclear Weapons. Oxford Research Group, Oxford.

[6] International Atomic Energy Agency (1988) The Radiological accident in Goiânia. International Atomic Energy Agency, Vienna.

[7] Johnson, D. (2017) Certified Training for Nuclear and Radioactive Source Security Management. Radiation Protection Dosimetry, 173, 87-92. https://doi.org/10.1093/rpd/ncw313

[8] Valentin, J. (2005) Protecting People against Radiation Exposure in the Event of a Radiological Attack. A Report of The International Commission on Radiological Protection. Annals of the ICRP, 35, 1-110, iii-iv. 